\title{
An adjustable table tennis bat and grip system for tetraplegics
}

\author{
DM Taktak \\ Division of Sport Science, the Manchester Metropolitan University, UK
}

\begin{abstract}
A table tennis bat and gripping system has been devised which can be used by people who are tetraplegic who are unable to hold the bat unassisted. The multi-positional head of the bat allows the user to alter the bat angle with respect to the hand whilst an elasticated glove ensures the bat is held firmly in place allowing the player access to a greater range of playing shots. The system has been used successfully to introduce recently injured players to the game, and has also been used by a British ranked tetraplegic table tennis player. It is suitable for general, rehabilitative or competition use.
\end{abstract}

Keywords: tetraplegia; table tennis and rehabilitation

\section{Introduction}

Table tennis has become a popular sport among those who are paraplegic or tetraplegic, and has often been employed as a useful remedial exercise during hospital treatment. ${ }^{1}$ Once discharged from hospital, many players continue the sport as a recreational activity, some playing competitively at club or national level.

Tetraplegic individuals with lesions below C6 and C7 or Class II as defined by Strohkend ${ }^{3}$ are unable to hold the bat unaided, due to paralysis of the muscles of their finger and thumb, although many are able to play table tennis provided the deltoid, biceps and triceps are functioning. Tetraplegic players of Class $\mathrm{I}^{3}$ are also able to play table tennis even though the triceps is paralysed, by using a modified service. ${ }^{1}$ It has been seen, however, that by adopting intensive training, such players have succeeded in beating those who are paraplegic with normal function of all upper musculature.

The conventional method of attaching the bat to the hand is by using a bandage, firmly strapping the player's fingers around the handle of the bat providing the necessary gripping force to allow the bat to stay firmly in place for the duration of the game..$^{1,2}$ However, the application of such strapping is very time consuming and does not allow the player to release the pressure applied around the hand during a match. Consequently the circulation may be restricted for a significant period and the player cannot inspect the hand regularly for any adverse rubbing to the skin, a problem if there is impaired sensation.

A previous attempt was made by Slatter and $\mathrm{Gibb}^{2}$ to develop a glove that held a standard table tennis

Correspondence: DM Taktak, B. Eng (Hons) in Mechan Engineering; Ph.D., Division of Sport Science, Crewe + Alsager Faculty, The Manchester Metropolitan University, Hassall Road, Alsager, Cheshire, ST7 2HL, UK bat in the hand of those who are tetraplegic. This was an adaptation of a wheelchair pusher glove, a leather glove incorporating Velcro fastenings and had proved successful in its usage.

\section{Problems encountered}

With the bat held firmly in the hand, the type and range of shots which can be played is severely limited. Once strapped in place, there can be little movement in the bat head relative to the rest of the arm. Where the player adopts a conventional style of play, problems have been encountered in the forearm shots, particularly those when the elbow is close to the body, where it is difficult for the player to angle the bat such that the ball crosses the net. It was therefore the initial aim of this study to try to correct for this in an alternative bat design.

\section{Design of the table tennis bat}

An attempt was first made to discover whether any commercial bats for those who are paraplegic or tetraplegic had been developed. Other than the conventional design, the search revealed only one bat in which the handle had been altered to incorporate a pistol grip design. This bat was also constructed with a fixed head, set at approximately $30^{\circ} \mathrm{C}$ as shown in Figure 1, but included a handle which encompassed the index and second fingers to assist in the grip of the bat.

The initial idea for this new bat was to look at the range of different bat head angles which would be required to combat the problems encountered by players in their forearm shots. The study focused initially on a single player who was experiencing such a difficulty. He was tetraplegic (C6 level, or Class II $^{3}$ ) who was a ranked British table tennis player. The 


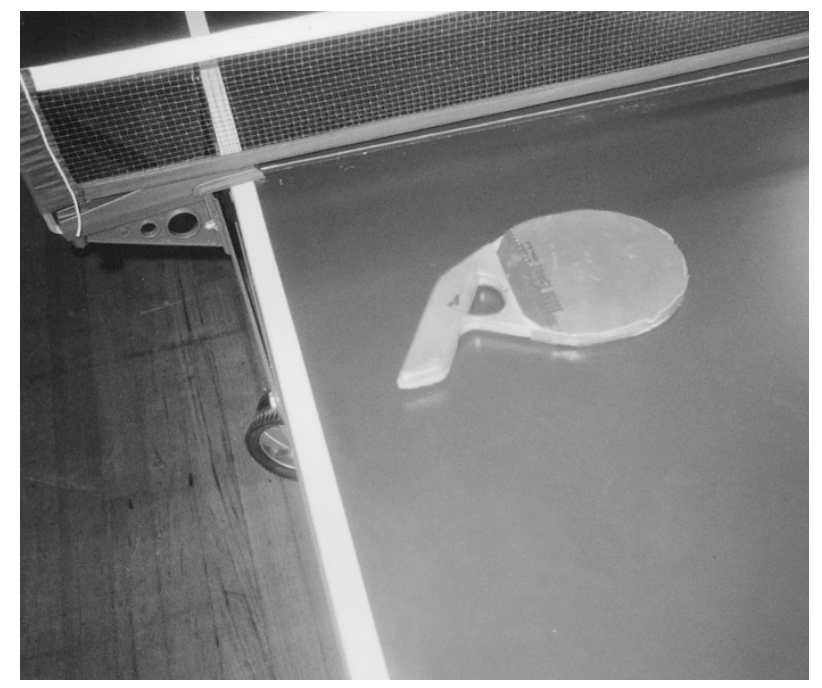

Figure 1 Pistol grip bat

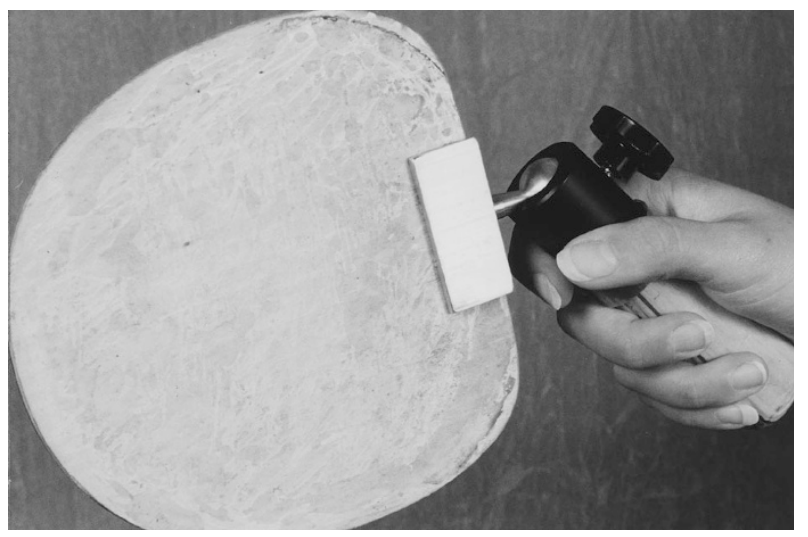

Figure 2 Adjustable table tennis bat

player required aid in gripping a table tennis bat and had sufficient elbow extension and hand flexion, though without normal power.

From video data obtained from the subject during play, it could be seen that to obtain a successful forehand shot, an angle was required which would incline the bat head in both the longitudinal and transverse planes through an angle varying between generally $0-20$ degrees in each plane. Thus, as the necessary inclination of the bat head varied for the subject, it was anticipated that for general use by all who are tetraplegic, the head would need to be capable of being fixed at any angle.

To facilitate this, a conventional table tennis bat was adapted to incorporate a ball and socket joint at the interface of the bat head and handle (ball and socket joint, Kaiser Fototechnik Ltd., Germany) (Figure 2). This joint could be locked at any angle

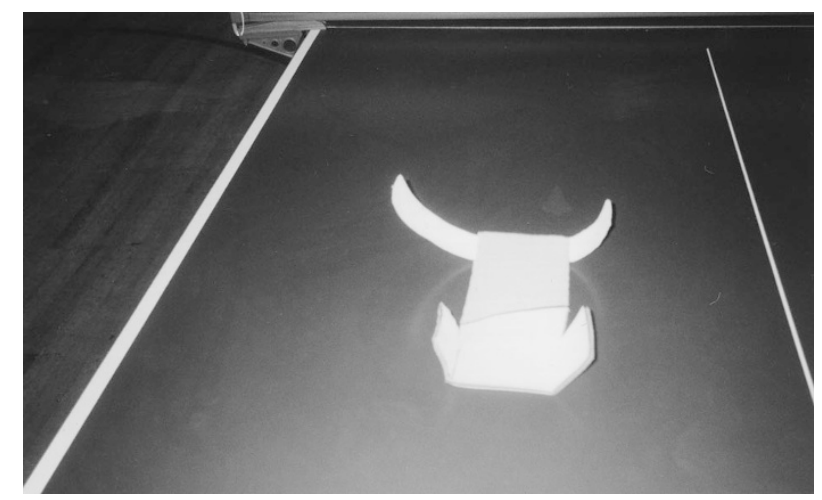

Figure 3 Elasticated gripping glove

by means of a standard screw which located into the base of the joint and which was adapted at the end to allow easy gripping to turn the screw. However, as this screw was close to the gripping area of the bat, it was elongated so as to avoid undue pressure on the skin.

A problem was encountered in fastening the joint to the bat head as the attachment left a raised area on the face of the bat. This, however, was compensated for, by applying a layer of foam between the wooden surface of the bat and the layer of rubber on the playing surface.

To attach the bat to the hand, a glove was made up using Neoprene (Smith and Nephew Ltd), (Figure 3), a slightly stretchable material to which Velcro is easily attached. The use of Velcro strapping thus meant that the glove would be suitable for a range of different hand sizes, and due to it being elasticated, this suggested that the glove would possibly alleviate some of the problems of impaired circulation, whilst still providing a firm grip onto the bat handle during play.

It was anticipated that if pressure problems persisted due to the gripping of the bat or gripping was more difficult for some individuals who are tetraplegic, the bat handle could be built up and moulded to suit the hand of the individual, thus making grip easier, and reducing any areas of higher pressure. The application of a heat setting putty around the handle could be an easy solution.

\section{Conclusion}

The bat has been used successfully for 12 months, although experimentation is obviously required before the appropriate positioning is obtained for optimum play by each individual.

Movement of the bat head relative to the hand has meant that a greater range of shots are now available to the player. This new flexibility may well help to mimic the ability of players with functional hand control of altering the position of the bat in the hand during the course of play. Whilst it is still difficult to 
change the bat head angle during the course of a game, it is possible to alter the angle in between games depending on the type of play and amount of spin required on the ball. This bat could also be used to experiment with a variety of bat head angles before construction of a custom made angled bat.

A possible improvement to the comfort of the bat would be to alter the screw adjustment arrangement to fit flush with the bat handle. This would then require a separate key to set the bat head angle that would have to be designed to be easily utilised by people with insufficient dexterity.

\section{Acknowledgements}

The author would like to thank staff at the Wrekin Area Scope Project, Telford, for their assistance and support.

\section{References}

1 Sir Ludwig Guttmann. Textbook of Sport for the Disabled. H.M\&M Publishers Ltd., Aylesbury, Bucks, UK, 1976.

2 Slatter ER, Gibb MM. Table Tennis Glove for Tetraplegics. Paraplegia, 1979; 17: 259-261.

3 Strohkendl H. Classification for Wheelchair Table Tennis. Palaestra, 1989; 5: 49-52. 